\title{
Brillouin scattering studies of acoustic phonon modes and central peak in single-crystal $\mathrm{Pb}\left(\mathrm{Sc}_{1 / 2} \mathrm{Ta}_{1 / 2}\right) \mathrm{O}_{3}$
}

\author{
V. Sivasubramanian ${ }^{1, *}$ and S. Kojima ${ }^{2, \dagger}$ \\ ${ }^{1}$ Condensed Matter Physics Division, Indira Gandhi Centre for Atomic Research, Kalpakkam-603102, India \\ ${ }^{2}$ Institute of Materials Science, University of Tsukuba, Tsukuba, Ibaraki 305-8573, Japan \\ (Received 27 January 2010; revised manuscript received 17 January 2012; published 8 February 2012)
}

\begin{abstract}
Brillouin spectra measurements have been carried out from 800 to $100 \mathrm{~K}$ for $\mathrm{Pb}\left(\mathrm{Sc}_{1 / 2} \mathrm{Ta}_{1 / 2}\right) \mathrm{O}_{3}$ in back-scattering geometry. The frequency of the longitudinal acoustic (LA) phonon begins to show marked softening followed by an increase in its width below the intermediate temperature $T^{*}=450 \mathrm{~K}$. The gradual increase in the width of the LA phonon below $T^{*}$ is attributed to the electrostrictive coupling between the local polarization and strain. The frequency of the LA phonon mode exhibits anomalous behavior around $295 \mathrm{~K}$, the phase transition temperature. The width of the LA phonon exhibits a sharp Landau-Khalatnikov-like maximum at $295 \mathrm{~K}$. A central peak begins to appear around $T^{*}$ and exhibits critical slowing down upon further cooling. The relaxation times calculated for the LA phonon and central peak indicate the order-disorder nature of the phase transition.
\end{abstract}

DOI: 10.1103/PhysRevB.85.054104

PACS number(s): 77.84.-s, 62.65.+k, 78.35.+c

\section{INTRODUCTION}

Relaxor ferroelectrics (RFE) are characterized by a broad and frequency-dependent maximum in the dielectric susceptibility as a function of temperature, broad distribution, and divergence of relaxation time on cooling with no macroscopic change in crystal structure. ${ }^{1}$ The origin of the complex behavior exhibited by RFEs is found to be due to the nucleation and growth of the polar nanoregions (PNRs) that begin to appear at a temperature, the so called Burns temperature $T_{B}{ }^{2}{ }^{2}$ Extensive studies on the dynamical aspects of PNRs have been carried out in prototype RFEs such as $\mathrm{Pb}\left(\mathrm{Mg}_{1 / 3} \mathrm{Nb}_{2 / 3}\right) \mathrm{O}_{3}$ $(\mathrm{PMN})$ and other related systems through neutron and light scattering studies. ${ }^{3-9}$ Brillouin scattering studies in these systems directly revealed the dynamical features of the PNRs such as the slowing down of their relaxation time and the coupling between the polarization fluctuations and acoustic phonon modes through the appearance of central peak (CP) and softening of the acoustic phonon mode. ${ }^{6-9}$

This paper focuses on micro-Brillouin scattering studies on the dynamical aspects of PNRs in partially ordered $\mathrm{Pb}\left(\mathrm{Sc}_{1 / 2} \mathrm{Ta}_{1 / 2}\right) \mathrm{O}_{3}$ (PST) without any $\mathrm{Pb}$ vacancies. PST-type compounds exhibit varied ferroelectric behavior depending on the degree of $B$-site ordering. Quenched PST exhibits a random distribution of $B$-site cations and shows a relaxor-type ferroelectric behavior whereas the annealed one shows a long-range ordering of $B$-site ions and exhibits normal ferroelectric behavior. ${ }^{10} \mathrm{~A}$ spontaneous relaxor-to-ferroelectric phase transition accompanied by a change in the structure from cubic to rhombohedral below $T_{m}$, the temperature of maximum dielectric constant has also been observed in these systems. ${ }^{11-13}$ Raman and IR spectroscopic studies of PST showed the increase in intensity and narrowing of the width of the phonon modes with increase in $B$-site chemical ordering. ${ }^{14,15}$ The presence of $\mathrm{Pb}$ vacancies also strongly influences the structural and dielectric behavior of PST. It has been found that in disordered PST with Pb vacancies (PST-DV) the spontaneous relaxor-ferroelectric transition is suppressed and the compound shows only relaxor behavior. The dielectric response of PST-DV is also considerably reduced and $T_{m}$ shifts to a lower temperature with respect to that without
$\mathrm{Pb}$ vacancies. ${ }^{11}$ Brillouin light scattering studies on PST-DV showed broad anomalies of longitudinal acoustic (LA) phonon modes at $T_{m}$ and a central peak (CP). ${ }^{16}$ Since the degree of $B$-site chemical ordering and the presence of $\mathrm{Pb}$ vacancies influence the ferroelectric behavior, it is also expected to influence the behavior of acoustic phonon modes and the CP. The aim of the present work is to study the anomalous behavior of acoustic phonon and $\mathrm{CP}$ across the phase transition by Brillouin light scattering for a partially ordered PST single crystal without any $\mathrm{Pb}$ vacancies.

\section{EXPERIMENT}

Single crystals of PST were grown by a flux method as described elsewhere. ${ }^{17}$ To determine the degree of $B$-site ordering ( $S$ parameter), powder x-ray diffraction was carried out using Philips X'Pert MPD diffractometer on the crushed single crystals. Dielectric measurements were carried out using a digital LCR meter in the temperature range 100-450 K. Brillouin light scattering measurements were performed on the (100) surface of the crystal in back-scattering geometry using a high-contrast $3+3$ pass Sandercock tandem Fabry-Perot interferometer. A diode-pumped solid-state laser (Coherent Compass 532) of wavelength $532 \mathrm{~nm}$ was used as an excitation source. Measurements were carried out in the FSR (Free Spectral Range) ranges of 75 and $400 \mathrm{GHz}$ in the temperature range of 800-100 K using a Linkam (THMS 600) cryostat.

\section{RESULTS AND DISCUSSION}

The powder x-ray diffraction pattern of PST is shown in Fig. 1. The super-structure peak at $2 \theta=19^{\circ}$ corresponds to the $B$-site ordering is clearly observed. The degree of $B$-site ordering $S=\left[\left(I_{111} / I_{200}\right)^{1 / 2}\right]_{\exp } /\left[\left(I_{111} / I_{200}\right)^{1 / 2}\right]_{\text {theor }}$ was calculated from the comparison of the integrated intensities of the super-structure (111) and the adjacent fundamental (200) reflections following a method used for PST ceramics. ${ }^{18}$ For completely ordered PST, the theoretical intensity ratio $I_{111} / I_{200}$ is $1.33 .^{18}$ The degree of $B$-site ordering of as-grown single crystals is found to be $S=0.55$. The average size 


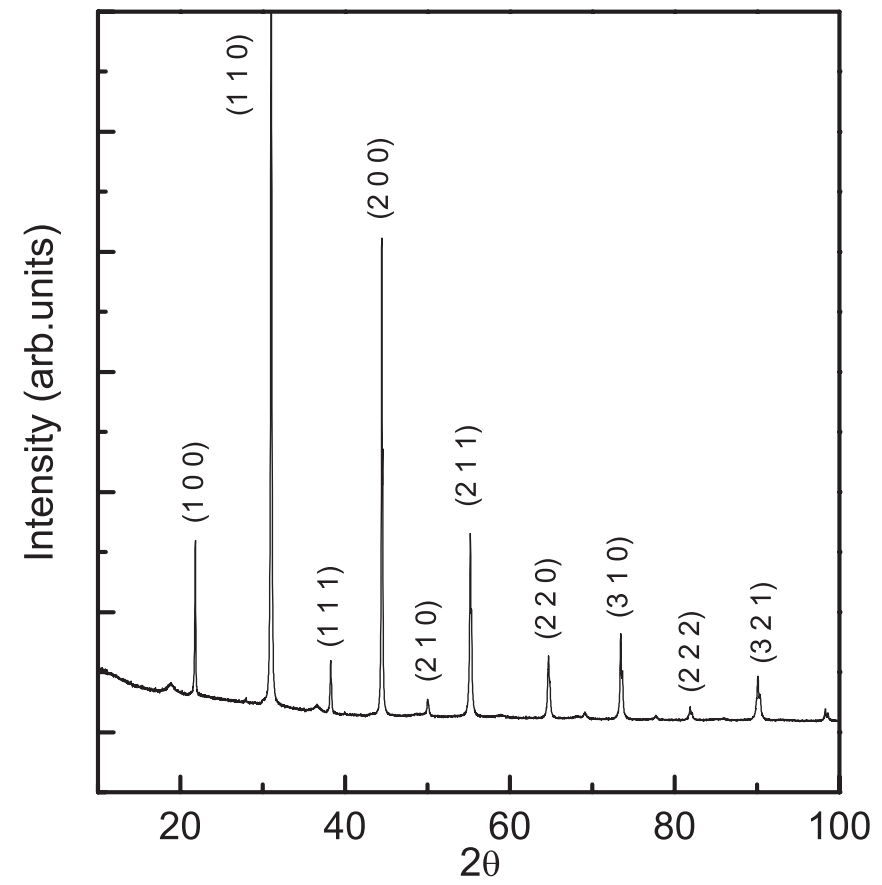

FIG. 1. Powder x-ray diffraction pattern of PST.

of the ordered domains calculated from the width of the super-structure reflection is about $10 \mathrm{~nm}$. The low-frequency dielectric dispersion shown in Fig. 2 indicates that PST exhibits a weak relaxor-like behavior. The value of $T_{m}$, the temperature at which the dielectric constant reaches maximum obtained from the dielectric measurements, is $297 \mathrm{~K}$. The X-ray diffraction studies for the similarly ordered PST show that at $T_{m}$ the structure changes from cubic to rhombohedral. ${ }^{13}$ Figure 3 shows the typical Brillouin spectra of PST in the FSR

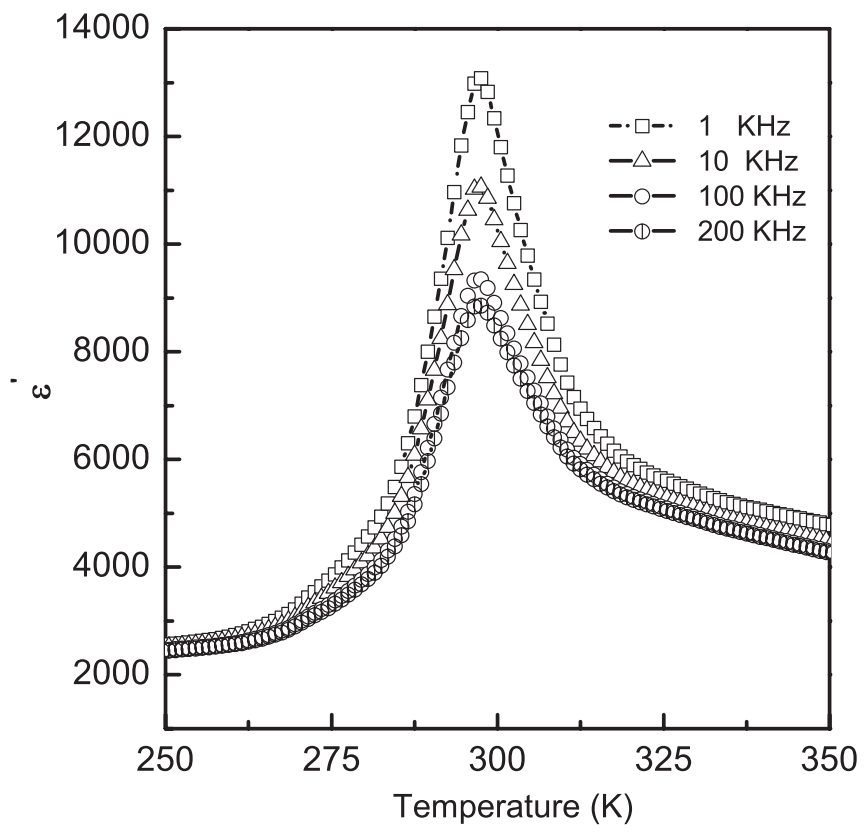

FIG. 2. Temperature dependence of real $\left(\varepsilon^{\prime}\right)$ part of dielectric constant of PST.

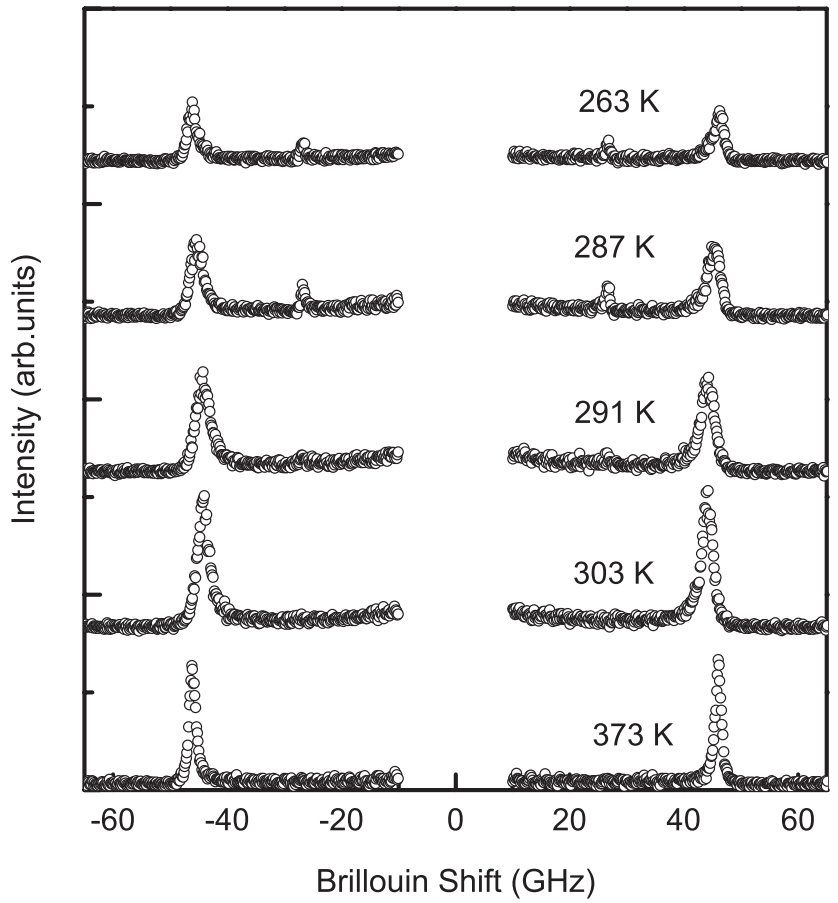

FIG. 3. Brillouin spectra of PST measured in the FSR range of $75 \mathrm{GHz}$ at some selected temperatures.

range of $75 \mathrm{GHz}$ at some selected temperatures. The spectra were fitted with the Voigt function with the fixed Gaussian width to account for the instrumental broadening. Above $295 \mathrm{~K}$, only the LA phonon mode could be observed at the back-scattering geometry. A transverse acoustic (TA) phonon mode appears below $295 \mathrm{~K}$. However, the TA phonon mode does not exhibit noticeable temperature dependence below $T_{m}$ and hence will not be discussed further. The temperature dependence of Brillouin shift of the LA phonon mode and its width (FWHM) are shown in Fig. 4. Above $600 \mathrm{~K}$, the Brillouin shift and FWHM of the LA phonon mode is almost constant independent of temperature. Since no interaction is expected to exist between the LA phonon and other degrees of freedom in this high-temperature range, the temperature dependence of LA phonon is mainly governed by the lattice anharmonicity. Below $600 \mathrm{~K}$, the LA phonon mode begins to deviate from the normal high-temperature behavior and exhibits marked softening with decrease in temperature. Further, the frequency of the LA phonon exhibits a sharp minimum at $295 \mathrm{~K}$, the transition temperature. As shown in Fig. 4, the width of the phonon mode begins to show a gradual increase at $450 \mathrm{~K}$ with a sharp maximum at the transition temperature. The behavior of the LA phonon mode in the present study is in contrast to PST-DV, ${ }^{16}$ where the relaxor behavior is retained due to the suppression of the spontaneous structural phase transition. In PST-DV, the relaxor characteristics are also clearly manifested in the broad anomalous features of the LA phonon across $T_{m}$ and also in the absence of the TA mode throughout the temperature range measurement. In the present study, very sharp anomalies of the LA phonon at $T_{m}$ followed by the appearance of the TA mode below $T_{m}$ clearly reveal the occurrence of the phase transition from cubic to rhombohedral structure. 


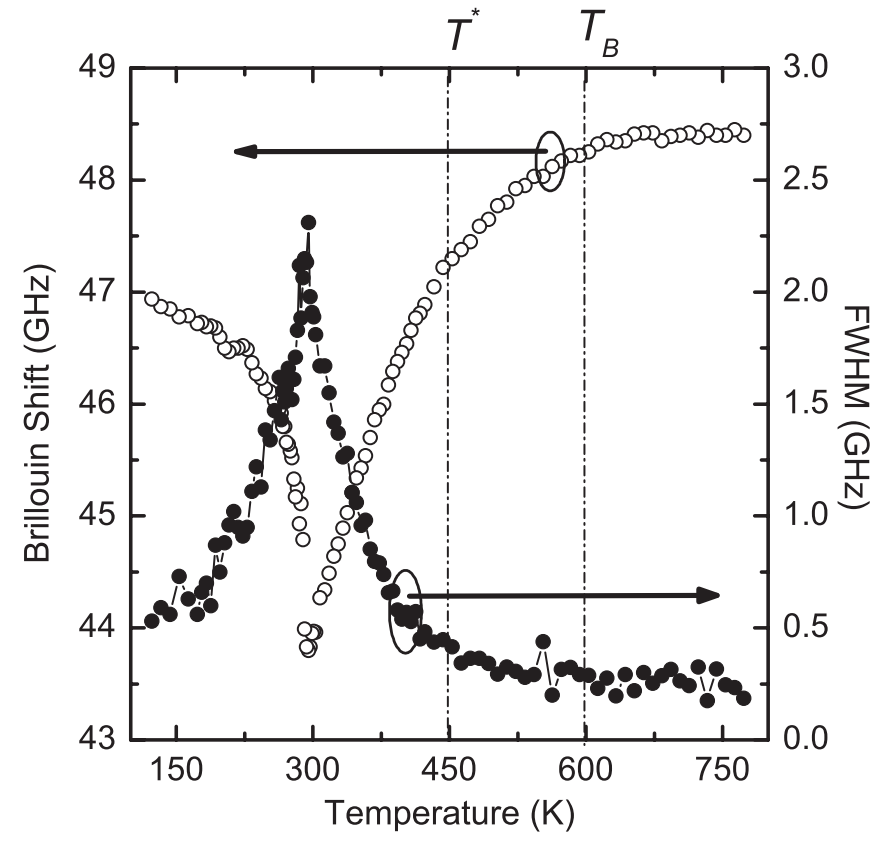

FIG. 4. Temperature dependences of Brillouin shift (open circles) and the width (FWHM) (closed circles) of the longitudinal acoustic phonon mode of PST.

As can be seen from Fig. 4, the gradual softening of the frequency followed by the gradual increase in the width of the LA phonon far above $T_{m}$ reveals that the order parameter fluctuations (polarization fluctuation) are the dominant dynamic mechanism. The dynamic fluctuation contribution is a characteristic of a $Q^{2} \varepsilon$-type quadratic coupling (electrostriction), squared in order parameter and linear in strain. The expression for the free energy density in the Landau theory of phase transition has the following three parts: ${ }^{19}$

$$
F\left(Q_{i}, \varepsilon_{k}, T\right)=F_{n}\left(Q_{i}\right)+F_{m}\left(\varepsilon_{k}\right)+F_{c}\left(Q_{i}, \varepsilon_{k}\right) .
$$

The first term is the free energy associated with the order parameter $Q_{i}$, i.e., polarization $P$ in the present case, the second term is the free energy associated with the strain $\varepsilon_{k}$, and the last term is the free energy of the interaction between the order parameter and strain. The coupling term $F_{c}$ is usually written in terms of increasing powers of $\varepsilon_{k}$ and $Q_{i}$ as

$$
F_{c}\left(\varepsilon_{i}, Q_{k}\right)=\beta_{i j} Q_{i} \varepsilon_{j}+\gamma_{i j k} Q_{i} Q_{j} \varepsilon_{k}+\delta_{i j k} Q_{i} \varepsilon_{j} \varepsilon_{k}+\cdots .
$$

The first term is the bilinear coupling between the order parameter and strain while the second term corresponds to the quadratic coupling between the order parameter and the strain. The effective elastic constant due to the change in polarization, driven by the change in strain is given by

$$
C_{i j}=C_{i j}^{\infty}-\sum_{k, l} \frac{\partial^{2} F_{c}}{\partial \varepsilon_{m} \partial Q_{k}}\left(\frac{\partial^{2} F}{\partial Q_{k} \partial Q_{l}}\right)^{-1} \frac{\partial^{2} F_{c}}{\partial \varepsilon_{n} \partial Q_{l}} .
$$

Since there is no macroscopic spontaneous polarization $(\langle P\rangle=0)$ owing to the random orientations of PNRs in the relaxor phase of PST, one can expect the quadratic coupling between the order parameter and strain to be the dominant in the coupling free energy $F_{c}$. The change in elastic constant for quadratic coupling can be written as ${ }^{19}$

$$
\Delta C=C-C^{\infty}=\gamma^{2}\left\langle P^{2}\right\rangle \chi^{(\varepsilon)},
$$

where $\left\langle P^{2}\right\rangle$ is the square of the local polarization, $\gamma$ is the electrostrictive coefficient and $\chi^{(\varepsilon)}$ is the clamped susceptibility for the order parameter. The classical Landau theory for second-order phase transition predicts only a steplike anomaly for $C(T)$ at the phase transition temperature. Therefore Landau theory cannot explain the significant softening of the LA phonon between $600 \mathrm{~K}$ and $T_{m}$. In RFEs, however, the local polarizations appear at $T_{B}$ and gradually grow with decrease in temperature. Although the average polarization is zero, their appearance will contribute to the decrease in the elastic constant due to the quadratic coupling $\left(\left\langle P^{2}\right\rangle \neq 0\right)$. In the prototype RFEs such as PMN and PMT indeed the elastic constant begins to exhibit a gradual decrease below $T_{B}{ }^{20,21}$ In these systems, the decrease in the elastic constant from $T_{B}$ evolves continuously with a broad minimum at $T_{m}$. For PST also, as in prototype relaxors, quadratic coupling between the PNRs and strain is clearly manifested with the gradual decrease in the frequency of the LA phonon at $T_{B}=600 \mathrm{~K}$ followed by increase in its width notably from $450 \mathrm{~K}$ as shown in Fig. 4. Note that the structural phase transition in PST is accompanied by the spontaneous transition from relaxor to normal ferroelectric phase. Normal ferroelectric state in PST is characterized by long-range ferroelectric order with nonvanishing macroscopic spontaneous polarization $(\langle P\rangle \neq 0)$ along $\langle 111\rangle$ direction. $^{12}$ In this case, one would expect the first term, i.e., bilinear coupling, linear in order parameter (polarization) and strain to be the dominant term in the coupling free energy $F_{c}$. Therefore the sharp damping maximum for the LA phonon at $295 \mathrm{~K}$ can evidently be connected with the Landau-Khalatnikov-like relaxation mechanism, which is associated with the bilinear coupling. The behavior of the width of the LA phonon in the present study is very similar to that is observed in $\mathrm{Rb}_{1-x}\left(\mathrm{ND}_{4}\right)_{x} \mathrm{D}_{2} \mathrm{AsO}_{4}$ with $x=0.10$ and $\mathrm{Pb}\left(\mathrm{Zn}_{1 / 3} \mathrm{Nb}_{2 / 3}\right) \mathrm{O}_{3}$ (PZN)-9.5\%PT. ${ }^{22,23}$ Both these systems exhibit quadratic-type coupling far from the transition temperature and show a weaker Landau-Khalatnikov-type maximum at the transition temperature. The relaxation time $\tau_{\mathrm{LA}}$ related to the LA phonon below $T_{m}$ can be calculated assuming a single relaxation time, from the following relation: ${ }^{19,24,25}$

$$
\frac{1}{2 \pi \tau_{\mathrm{LA}}}=\frac{v_{\infty}^{2}-v^{2}(T)}{\Gamma(T)-\Gamma_{\infty}}
$$

where $v(\Gamma)$ is the observed Brillouin shift (width) at a given temperature and $v_{\infty}\left(\Gamma_{\infty}\right)$ is the Brillouin shift (FWHM) not affected by the structural phase transition. The constant values of $v$ and $\Gamma$ in the high-temperature limit is used for $v_{\infty}$ $(48.5 \mathrm{GHz})$ and $\Gamma_{\infty}(0.27 \mathrm{GHz})$. The temperature dependence of the inverse relaxation time $\left(1 / \tau_{\mathrm{LA}}\right)$ related to LA phonon as shown in Fig. 5 exhibits approximately a linear behavior. Such a behavior is known as "critical slowing down" where $1 / \tau_{\text {LA }}$ is found to satisfy the following relation: ${ }^{25,26}$

$$
\frac{1}{\tau_{\mathrm{LA}}}=\frac{T_{m}-T}{T_{m}} \frac{1}{\tau_{o}}+\frac{1}{\tau_{1}} .
$$

Best fit to the experimental data with Eq. (6) yields $\tau_{o}=$ $0.22 \mathrm{ps}$ and $\tau_{1}=1.68 \mathrm{ps}$. The relaxation time $\tau_{o}$ for PST is of 


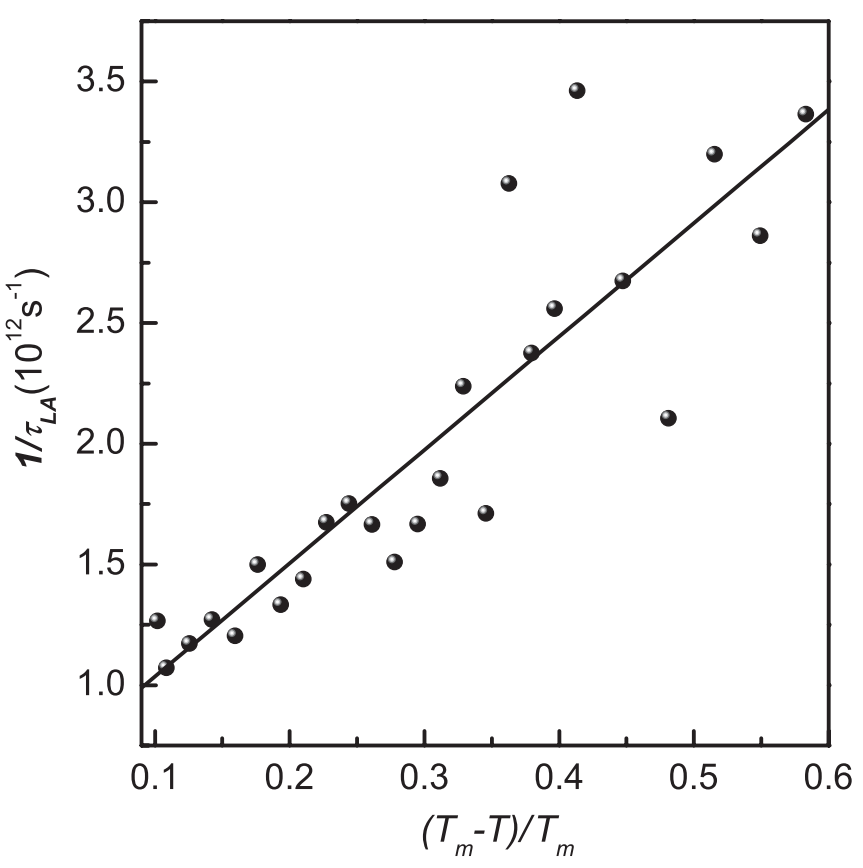

FIG. 5. $1 / \tau_{\mathrm{LA}}$ as a function of reduced temperature $\left(T_{m}-T\right) / T_{m}$ for the LA phonon; points are the experimental data and the solid line is the fit of Eq. (6).

similar order of magnitude as the relaxation time observed for typical order-disorder ferroelectrics. For instance, $\tau_{o}$ is $0.13 \mathrm{ps}$ for $\mathrm{KH}_{2} \mathrm{PO}_{4}$ (Ref. 27) and 0.10 ps for triglycine sulfate. ${ }^{28}$

In the FSR range of $400 \mathrm{GHz}$ as shown in Fig. 6, a broad relaxation mode (quasi-elastic scattering or central peak) with the marked temperature dependence is clearly observed around

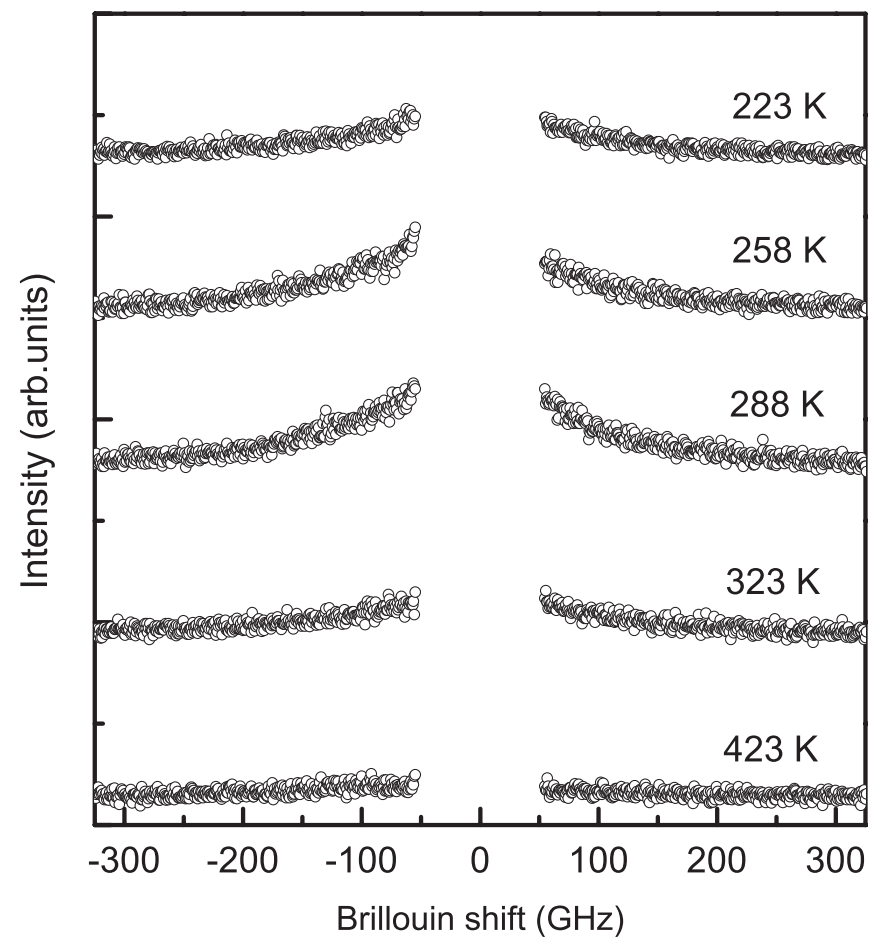

FIG. 6. Brillouin spectra of PST measured in the FSR range of $400 \mathrm{GHz}$ at some selected temperatures.

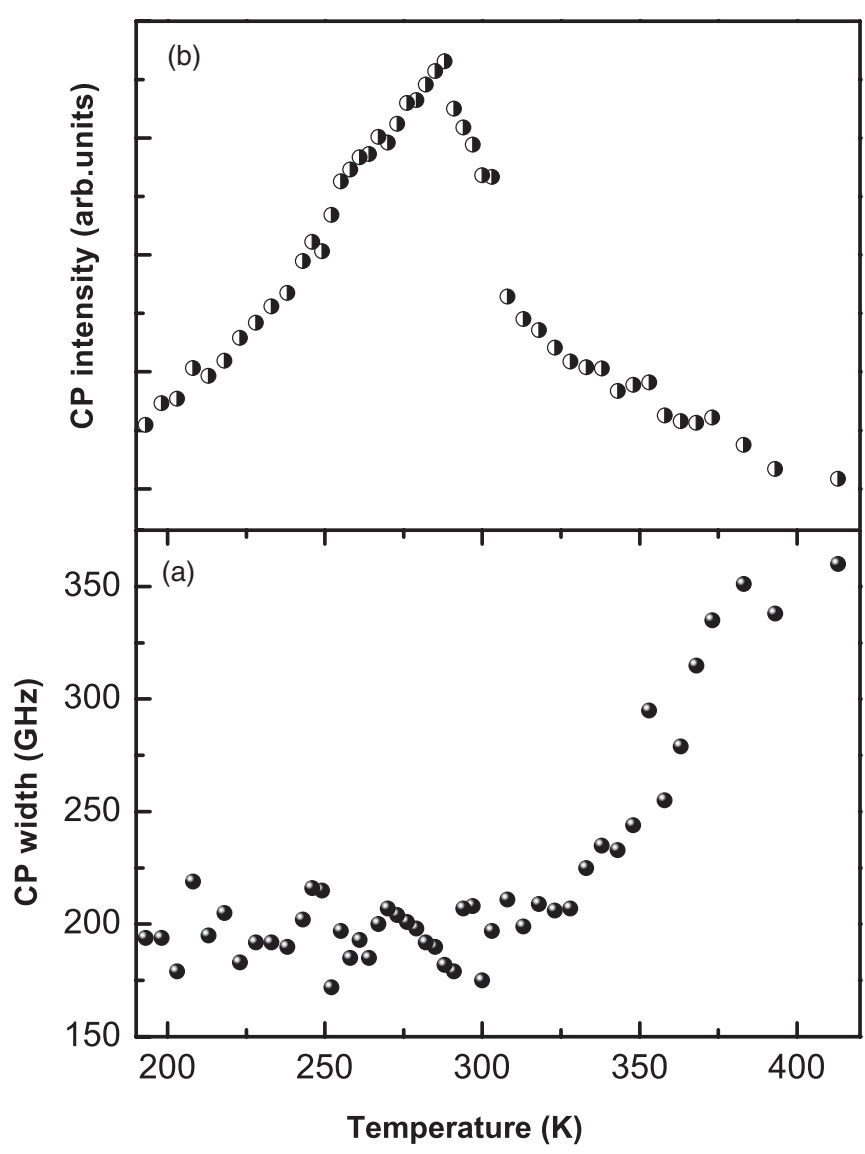

FIG. 7. (a) Temperature dependence of the width of the central peak. (b) Temperature dependence of the integrated intensity of the central peak.

the Raleigh tail. The CP begins to appear at about $450 \mathrm{~K}$ and its intensity increases further upon cooling. Assuming a Debye relaxation process, $\mathrm{CP}$ could be independently fitted with a single Lorentzian function centered at zero frequency. The temperature dependence of the width and the integrated intensity of the CP are shown in the Figs. 7(a) and 7(b). A decrease of the width of the CP followed by an increase in the intensity upon cooling towards $T_{m}$ is observed. In relaxor ferroelectrics, CP that begins to appear well above $T_{m}$ is attributed to the relaxational dynamics of PNRs.${ }^{5,7}$ As shown in Fig. 7, the decrease in the width and increase in the integrated intensity of $\mathrm{CP}$ indicates critical slowing down of the relaxation time and increase in the correlations between the PNRs with decrease in temperature. The ratio of the temperature to the integrated intensity of CP, $T / I_{c p}\left(I_{c p}\right.$ is the intensity of the central peak) is proportional to the inverse susceptibility as given below: ${ }^{29}$

$$
\begin{aligned}
T / I_{c p} & \propto \int_{0}^{\infty} \frac{\chi^{\prime \prime}(\omega)}{\omega} d \omega \propto \chi^{\prime}(0)^{-1}, \\
\chi(0)^{-1} & =\frac{T-T_{o}}{C}\left(T>T_{c}\right) .
\end{aligned}
$$

As shown in Fig. 8, the linear fit of $T / I_{c p}$ above $T_{m}$ yields $T_{o}=267 \mathrm{~K}$. Using the value of $T_{o}$ obtained from Eq. (7), the 


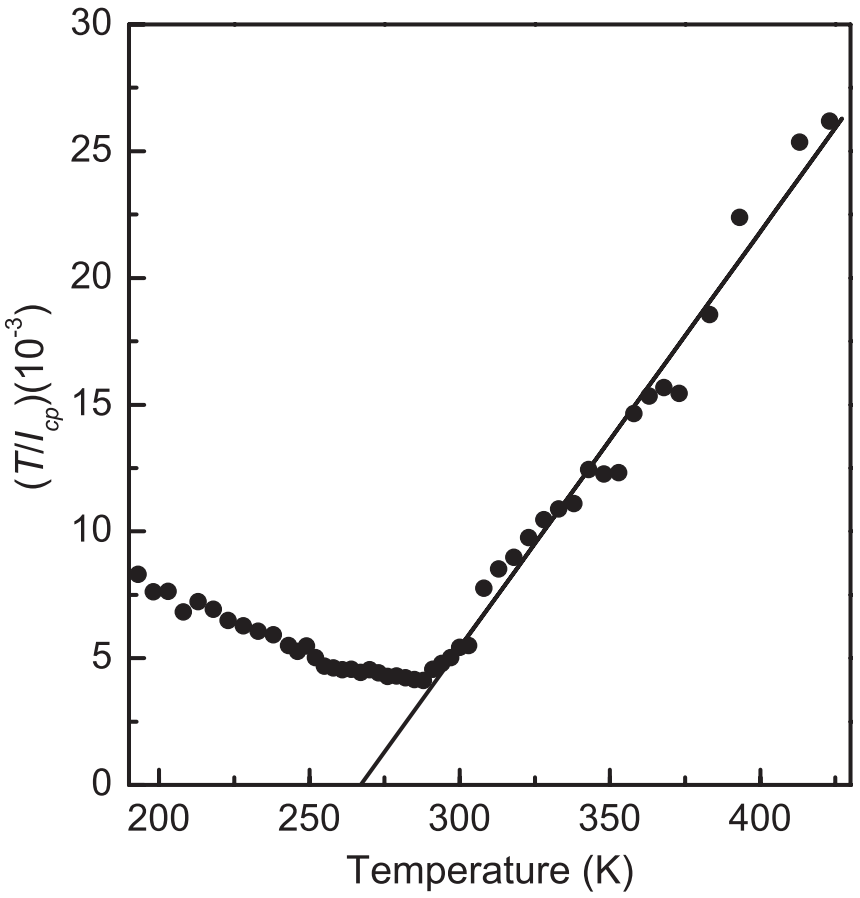

FIG. 8. Temperature variation of $T / I_{c p}$; points are the experimental data and the solid line is the fit of Eq. (7).

linear fit of the relation

$$
\frac{1}{\tau_{c p}}=\frac{T-T_{o}}{T_{o}} \frac{1}{\tau^{*}}+\frac{1}{\tau_{2}}
$$

in the vicinity of the phase transition is shown in Fig. 9. $\tau_{c p}$ is the relaxation time of the polarization fluctuations obtained from the central peak. The best fit of the experimental data yields $\tau^{*}=2.3 \mathrm{ps}$ and $\tau_{2}=7.7 \mathrm{ps}$. The value of $\tau^{*}$ is similar to that reported for $\mathrm{KD}_{2} \mathrm{PO}_{4}(1.3 \mathrm{ps}$ for $T>$ $\left.T_{c}\right)^{30}$ which exhibits order-disorder behavior in the vicinity of the transition temperature. A similar value is reported for disordered $\mathrm{Pb}\left(\mathrm{Sc}_{1 / 2} \mathrm{Nb}_{1 / 2}\right) \mathrm{O}_{3}$ (PSN) in the cubic phase $(5 \mathrm{ps}){ }^{31}$ These observations suggest that the $\mathrm{CP}$ originates from the polarization fluctuations and that an order-disorder mechanism contributes to the slowing down of the relaxation time.

Recent Raman and neutron scattering and acoustic emission studies have revealed more insights about the strong correlations between the local polarization of PNRs and strain. Based on the temperature evolution of the diffuse neutron and Raman scattering on PMN and PZN and PZN- $x$ PT with $x=0.045$, Toulouse et al. ${ }^{32}$ suggested the existence of another characteristic temperature between $T_{B}$ and $T_{m}$. Existence of this temperature $T^{*}$ was reflected in the anomalous variations in the intensity and the frequency of Raman optical modes and central peaks. $T^{*}$ has been suggested as the onset temperature of the development of strong correlations between the initially nucleated small dynamic PNRs. According to Toulouse et al..$^{32}$ the temperature range between $T_{B}$ and $T^{*}$ can be identified with the formation of short lived correlations between the off-centered ions, which results in the formation of small dynamic PNRs, while the range between $T^{*}$ and $T_{m}$ is characterized by the coupling between the adjacent PNRs

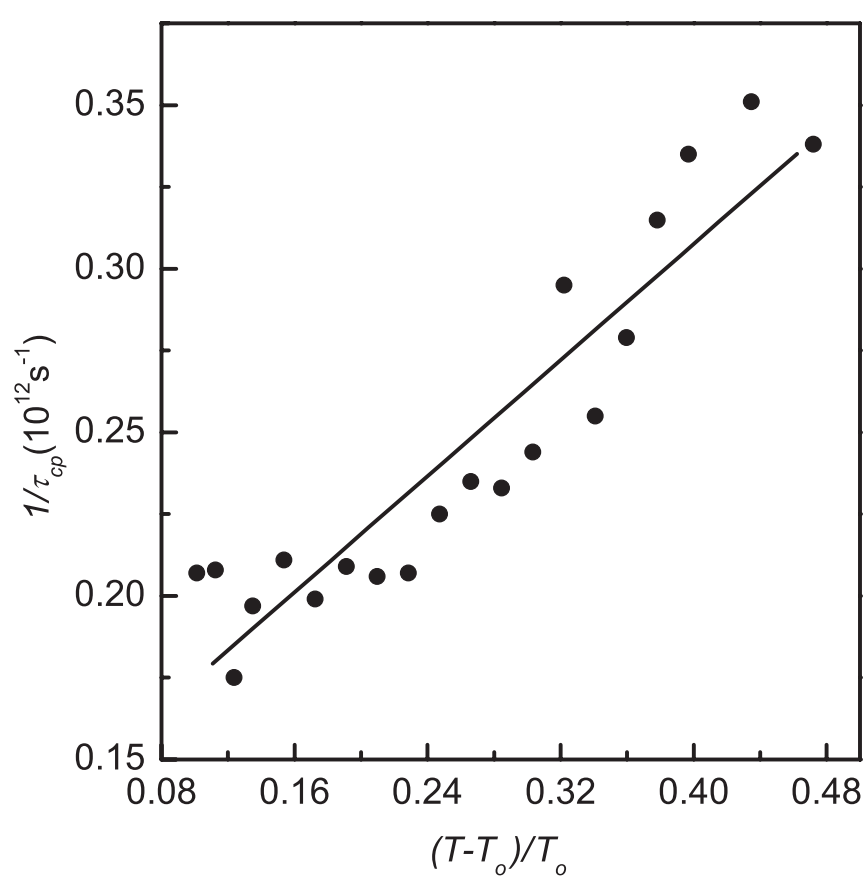

FIG. 9. $1 / \tau_{c p}$ as a function of reduced temperature $\left(T-T_{o}\right) / T_{o}$; points are the experimental data and the solid line is the best fit of Eq. (8) in the vicinity of $T_{m}$.

and their aggregation to form larger static PNRs. Acoustic emission studies in PZN and PZN-PT systems also confirmed the existence of this intermediate temperature $T^{*}$ and revealed that the corresponding local structural transformations are associated with the changes in the local strain fields. ${ }^{33,34}$ It has been demonstrated recently that $T^{*}$ is the same $(500 \pm 30 \mathrm{~K})$ for all $\mathrm{Pb}$-based relaxor ferroelectric systems. ${ }^{35} \mathrm{It}$ has also been shown through Brillouin scattering studies that in relaxor ferroelectric systems, significant softening of acoustic phonon and the appearance of $\mathrm{CP}$ begin to take place around $T^{*} \cdot{ }^{36} \mathrm{~A}$ recent study on the temperature evolution of phonon anomalies and unit cell parameter in PST and $\left(\mathrm{Pb}_{0.78} \mathrm{Ba}_{0.22}\right)\left(\mathrm{Sc}_{0.5} \mathrm{Ta}_{0.5}\right) \mathrm{O}_{3}$ revealed the existence of $T^{*}{ }^{37}$ For PST with the ordered domains of 6-nm size, the reported value of $T^{*}$ is $450 \mathrm{~K} .{ }^{33} \mathrm{In}$ the present study, since the average size of the ordered domains is about $10-\mathrm{nm}$, it can be expected that $T^{*}$ would be close to that reported for PST with 6-nm ordered domains. ${ }^{33}$ As shown in Fig. 2, an actual increase in the width of LA phonon at $450 \mathrm{~K}$ signifies the increase in the correlations between PNRs. In addition, an increase in the intensity of $\mathrm{CP}$ and a decrease in its width indicate that the density of PNRs and their interactions between them begin to increase at $450 \mathrm{~K}$. Therefore, in conjunction with Raman and x-ray diffraction studies, the temperature $450 \mathrm{~K}$ at which the width of the LA phonon begins to show a noticeable increase is identified with the intermediate temperature $T^{*}$. In the systems like PMN, PMT, as well as PST-DV, the strong correlations between PNRs does not lead to a macroscopic ferroelectric order whereas in PST, the strong correlations between PNRs that begin at $T^{*}$ lead to a long-range ordered dipolar state below transition temperature. In fact, the sharp damping maximum at the transition temperature is normally attributed to the rapid growth of long-range ferroelectric ordering. ${ }^{23}$ Consequently, PST 
exhibits crossover behavior from the quadratic-type coupling in the relaxor phase to the bilinear coupling in the ferroelectric phase.

\section{SUMMARY AND CONCLUSION}

We have found by Brillouin scattering in PST that the LA phonon exhibits a sharp anomaly across the phase transition from relaxor cubic to ferroelectric rhombohedral state. The significant increase in the damping of the LA phonon below $T^{*}=450 \mathrm{~K}$ is attributed to its electrostrictive coupling of the strain with the local polarizations of quasistatic PNRs. The cubic-rhombohedral structural phase transition at $295 \mathrm{~K}$ is evidenced from the sharp minimum in the frequency followed by the sharp maximum in the width of the LA phonon. The sharp damping maximum at $295 \mathrm{~K}$ is attributed to the Landau-Khalatnikov-like relaxation mechanism. The temperature dependence of $\mathrm{CP}$ exhibits a critical slowing down near the phase transition in the cubic phase. The relaxation time calculated for the LA phonon and the CP suggests the order-disorder nature of the phase transition.

\section{ACKNOWLEDGMENTS}

We thank S. Tsukada for useful suggestions and comments. We also thank J. Kano for his help in powder X-ray diffraction. One of the authors (V.S.) is grateful to the Japanese Society for Promotion of Science Invitation Fellowship Program for Research in Japan and to the Institute of Materials Science, University of Tsukuba, for hospitality. *shiva@igcar.gov.in

${ }^{\dagger}$ kojima@ims.tsukuba.ac.jp

${ }^{1}$ L. E. Cross, Ferroelectrics 76, 241 (1987).

${ }^{2}$ G. Burns and F. H. Dacol, Solid State Commun. 48, 853 (1983).

${ }^{3}$ S. G. Lushnikov, J.-H. Ko, and S. Kojima, Appl. Phys. Lett. 84, 4798 (2004).

${ }^{4}$ Y. Gorouya, Y. Tsujimi, M. Iwata, and T. Yagi, Appl. Phys. Lett. 83, 1358 (2003).

${ }^{5}$ J. H. Ko, S. Kojima, A. A. Bokov, and Z. G. Ye, Appl. Phys. Lett. 91, 252909 (2007).

${ }^{6}$ G. Shabbir and S. Kojima, Appl. Phys. Lett. 91, 062911 (2007).

${ }^{7}$ J. H. Ko, D. H. Kim, and S. Kojima, Appl. Phys. Lett. 90, 112904 (2007).

${ }^{8}$ J. H. Ko, D. H. Kim, S. Kojima, W. Chen, and Z. G. Ye, J. Appl. Phys. 100, 066106 (2006).

${ }^{9}$ S. Tsukada, Y. Ike, J. Kano, T. Sekiya, Y. Shimojo, R. Wang, and S. Kojima, J. Phys. Soc. Jpn. 77, 033707 (2008).

${ }^{10}$ N. Setter and L. E. Cross, J. Appl. Phys. 51, 4356 (1980).

${ }^{11}$ F. Chu, N. Setter, and A. K. Taganstev, J. Appl. Phys. 74, 5129 (1993)

${ }^{12}$ C. Malibert, B. Dkhil, J. M. Kiat, D. Durand, J. F. Berar, and A. Spasojevic-de Bire, J. Phys. Condens. Matter 9, 7485 (1997).

${ }^{13}$ P. M. Woodward and K. Z. Baba-Kishi, J. Appl. Cryst. 35, 233 (2002).

${ }^{14}$ U. Bismayer, V. Devarajan, and P. Groves, J. Phys.: Condens. Matter 1, 6977 (1989).

${ }^{15}$ I. M. Reaney, J. Petzelt, V. V. Voitsekhovskii, F. Chu, and N. Setter, J. Appl. Phys. 76, 2086 (1994).

${ }^{16}$ F. Jiang, J. H. Ko, S. G. Lushnikov, and S. Kojima, Jpn. J. Appl. Phys. 40, 5823 (2001).

${ }^{17}$ N. Setter and L. E. Cross, J. Cryst. Growth 50, 555 (1980).

${ }^{18}$ C. G. F. Stenger and A. J. Burggraaf, Phys. Status Solidi A 61, 275 (1980).

${ }^{19}$ W. Rehwald, Adv. Phys. 22, 721 (1973).
${ }^{20}$ S. G. Lushnikov, A. I. Fedoseev, S. N. Gvasaliya, J. H. Ko, and S. Kojima, J. Phys.: Condens. Matter 19, 496206 (2007).

${ }^{21}$ S. G. Lushnikov, A. I. Fedoseev, S. N. Gvasaliya, and S. Kojima, Phys. Rev. B 77, 104122 (2008).

${ }^{22}$ C. S. Tu and V. H. Schmidt, Phys. Rev. B 50, 16167 (1994).

${ }^{23}$ C.-H. Tu, F.-C. Chao, C.-H. Yeh, C.-L. Tsai, and V. H. Schmidt, Phys. Rev. B 60, 6348 (1999).

${ }^{24}$ S. Tsukada and S. Kojima, Phys. Rev. B 78, 144106 (2008).

${ }^{25}$ M. Mączka, J. Hanuza, A. Majchrowski, and S. Kojima, Phys. Rev. B 75, 214105 (2007).

${ }^{26}$ H. Z. Cummins and A. P. Levanyuk, Light Scattering Near Phase Transitions (North-Holland, Amsterdam, 1983).

${ }^{27}$ I. Taksuzaki, M. Kasahara, M. Tokunaga, and H. Tanaka, Ferroelectrics 39, 1049 (1981).

${ }^{28}$ R. W. Gammon and H. Z. Cummins, Phys. Rev. Lett. 17, 193 (1966).

${ }^{29}$ W. Hayes and W. Louden, Scattering of Light by Crystals (Dover, New York, 1978).

${ }^{30}$ R. L. Reese, I. J. Fritz, and H. Z. Cummins, Phys. Rev. B 7, 4165 (1973).

${ }^{31}$ M. Ahart, A. Husur, Y. Bing, Z. G. Ye, R. J. Hemley, and S. Kojima, Appl. Phys. Lett. 94, 142906 (2009).

${ }^{32}$ J. Toulouse, F. Jiang, O. Svitelskiy, W. Chen, and Z. G. Ye, Phys. Rev. B 72, 184106 (2005).

${ }^{33}$ E. Dul'kin, M. Roth, P. E. Janolin, and B. Dkhil, Phys. Rev. B 73, 012102 (2006).

${ }^{34}$ M. Roth, E. Mojaev, E. Dul'kin, P. Gemeiner, and B. Dkhil, Phys. Rev. Lett. 98, 265701 (2007).

${ }^{35}$ B. Dkhil, P. Gemeiner, A. Al-Barakaty, L. Bellaiche, E. Dul'kin, E. Mojaev, and M. Roth, Phys. Rev. B 80, 064103 (2009).

${ }^{36}$ J. H. Ko, D. H. Kim, and S. Kojima, Phys. Rev. B 77, 104110 (2008).

${ }^{37}$ B. Mihailova, B. Maier, C. Paulmann, T. Malcherek, J. Ihringer, M. Gospodinov, R. Stosch, B. Guttler, and U. Bismayer, Phys. Rev. B 77, 174106 (2008). 